\title{
Performance of extraction kicker magnet in a rapid cycling synchrotron
}

\author{
Junichiro Kamiya, ${ }^{*}$ Tomohiro Takayanagi, and Masao Watanabe \\ J-PARC Center, Japan Atomic Energy Agency, 2-4 Shirane Shirakata, Tokai-mura, Naka-gun, Ibaraki 319-1195, Japan
}

(Received 22 October 2008; published 23 July 2009)

\begin{abstract}
Installation of the kicker magnets in the extraction section of the rapid cycling synchrotron in the J-PARC facility has been completed. They succeeded in extracting the proton beam accelerated up to $3 \mathrm{GeV}$ in the first commissioning with beam. The operation parameters of the kicker system agreed well with the parameters, which were estimated from the magnetic field measurement and the current test of each power supply. The distribution of the integrated magnetic field is flat enough to meet the beam requirements. The beam based measurement of the flattop of the magnetic field was performed with the beam position monitors. Although a ringing structure was observed, which exceeded the specified limit, the degree of the flattop distortion could be corrected by the timing adjustment of the output current. The stability of the power supply is on the order of $\pm 0.2 \%$, which is sufficient to achieve stable beam extraction. The rise time of the output current largely depends on the gas pressure in the thyratrons. Dependence of the rise time on the gas pressure was strictly measured and the number of unwanted prefires could be reduced.
\end{abstract}

DOI: 10.1103/PhysRevSTAB.12.072401

PACS numbers: 29.20.dk, 29.27.Ac, 07.55.Db

\section{INTRODUCTION}

The Japan Proton Accelerator Research Complex (J-PARC) is a high-intensity proton accelerator facility project [1]. The accelerator complex comprises $181 \mathrm{MeV}$ linac (to be upgraded to $400 \mathrm{MeV}$ ), $3 \mathrm{GeV}$ rapid cycling synchrotron (RCS), and $50 \mathrm{GeV}$ synchrotron [main ring (MR)]. The RCS started its commissioning test with beam in September 2007. The beam was successfully accelerated by the rf system [2] and extracted by the kicker magnet system. The RCS is a keystone of the J-PARC facility because it assumes the role of both the main accelerator for a pulsed spallation neutron source facility and the injector to the MR. The RCS aims to achieve the proton beam power of $1 \mathrm{MW}$, which means each cycle two bunches of $8.3 \times 10^{13}$ protons are accelerated up to $3 \mathrm{GeV}$ at $25 \mathrm{~Hz}$ repetition rate. In order to extract such an unusual high power beam at the rapid repetition rate, there are several challenging requests for the kicker magnet system of the RCS. First, the rise time of the magnetic field should be fast enough to complete the generation of the requested magnetic field from the end of the first bunch passing through the kicker until the arrival of the head of the second bunch. The rise time has to be less than $357 \mathrm{~ns}$ according to the calculation results. In order to obtain such a fast rise time, we have to choose devices which are difficult to handle and operate in a complex configuration. For example, thyratrons, which are difficult to handle stably, have to be used as the switch for fast current rise time [3]. The distributed parameter type [4], which is more complex than the lumped parameter type, has to be chosen

\footnotetext{
*kamiya.junichiro@jaea.go.jp
}

for the magnet structure to reduce the transmission period in the magnet [5]. Second, the aperture of the magnet should be large in order to accept the high power beam with large emittance of $486 \pi \mathrm{mm}$ mrad. Therefore it is difficult to achieve a good uniformity of the magnetic field distribution. The improvement of the field distribution uniformity had been performed using a prototype kicker magnet [6]. The improvements were applied to the production magnets. Furthermore, the power supply has to provide a large excitation current due to the high gap aperture. The peak and average electrical power of the output reaches $90 \mathrm{MW}$ and $2.25 \mathrm{~kW}$, respectively. Therefore it became challenging to operate thyratrons in such a condition. The self-breakdown without trigger had happened for almost all the new thyratrons. It had been found that aging is needed for new tubes for operating in such a tough condition [6]. The third challenging point is the flattop of the magnetic field, which should be long enough to kick the two beam bunches. That is difficult to achieve because the flattop is disturbed by not only the impedance mismatching between the load cables and the magnet but also the induced magnetic field which originates from the magnet structure itself [7]. Finally, stable operation is strongly requested by the beam users. Stable operation means reproducibility both for short-term and the long-term time range. The short-term reproducibility is limited by the precision of the feedback circuit in the power supply. The long-term one is affected by the stability of the thyratrons. It is important to fix the reservoir voltage, at which the individual thyratron can be operated without self-breakdown while keeping a long lifetime. In this paper, we are reporting the specifications, performance, and the results of the improvements of the extraction kicker 
magnet of the RCS in J-PARC. In the next section, the design and specification of the kicker system is presented. In Sec. III the results of the magnetic field distribution for all eight kicker magnets are shown. The results of the flattop measurement based on the beam tests are shown in Sec. IV. The measurement result of improving the flattop length is also shown in the same section. The measurement results and the method for stable operation of the kicker system including the adjustment of the thyratron reservoir voltage are described in Sec. V. The summary is presented in Sec. VI.

\section{SPECIFICATIONS OF THE KICKER SYSTEM}

\section{A. System}

The summary of the specifications and measurement results in the R\&D phase had been reported in the previous paper [6]. In this section, we describe the detailed specifications of the production version of the kicker system. The specifications of the production version of the kicker system are summarized in Table I. There are in total eight kicker magnets at the extraction section in the RCS. There are three types of kicker magnets, S, M, and L, corresponding to their vertical gap height as shown in Table I. A proton beam is vertically defocused by a quadrupole magnet $\mathrm{Q}$ which is installed between the third and fourth kicker

TABLE I. Specifications of the RCS kicker system.

\begin{tabular}{lc}
\hline \hline Numbers & 8 (Nos. 1-8) \\
Maximum repetition rate & $25 \mathrm{~Hz}$ \\
Characteristic impedance & $10 \Omega$ \\
PFL & Coaxial cable, about $102 \mathrm{~m}$ \\
Load cable & Same as PFL, about 130 m \\
High power switch & Thyratron CX1193C, e2V Ltd. \\
Maximum output current & $4 \mathrm{kA}$ \\
Operation output current & $3 \mathrm{kA}$ \\
Flattop length & 840 ns for two beam bunches \\
Rise time & 25 ns (typical) \\
Jitter & Less than 10 ns \\
Flatness & $6 \%$ without correction \\
& $2 \%$ with correction \\
Maximum charging voltage & $80 \mathrm{kV}$ \\
Operation charging voltage & $60 \mathrm{kV}$ \\
Maximum exciting current & $8 \mathrm{kA}$ \\
Operation exciting current & $6 \mathrm{kA}$ \\
Magnet structure & Distributed parameter line \\
Magnet core & Ni-Zn ferrite PE14, TDK Ltd. \\
Magnet gap height & $153 \mathrm{~mm}$ (S-type: No. 3, 4, 5) \\
& 173 mm (M-type: No. 2, 6) \\
Magnet gap width & 199 mm (L-type: No. 1, 7, 8) \\
Magnet longitudinal length & $280 \mathrm{~mm}$ \\
Magnetic field & $638 \mathrm{~mm}$ \\
& $460 \mathrm{G} \mathrm{(S-type)}$ \\
\hline \hline & 360 G (L-type) \\
\hline
\end{tabular}

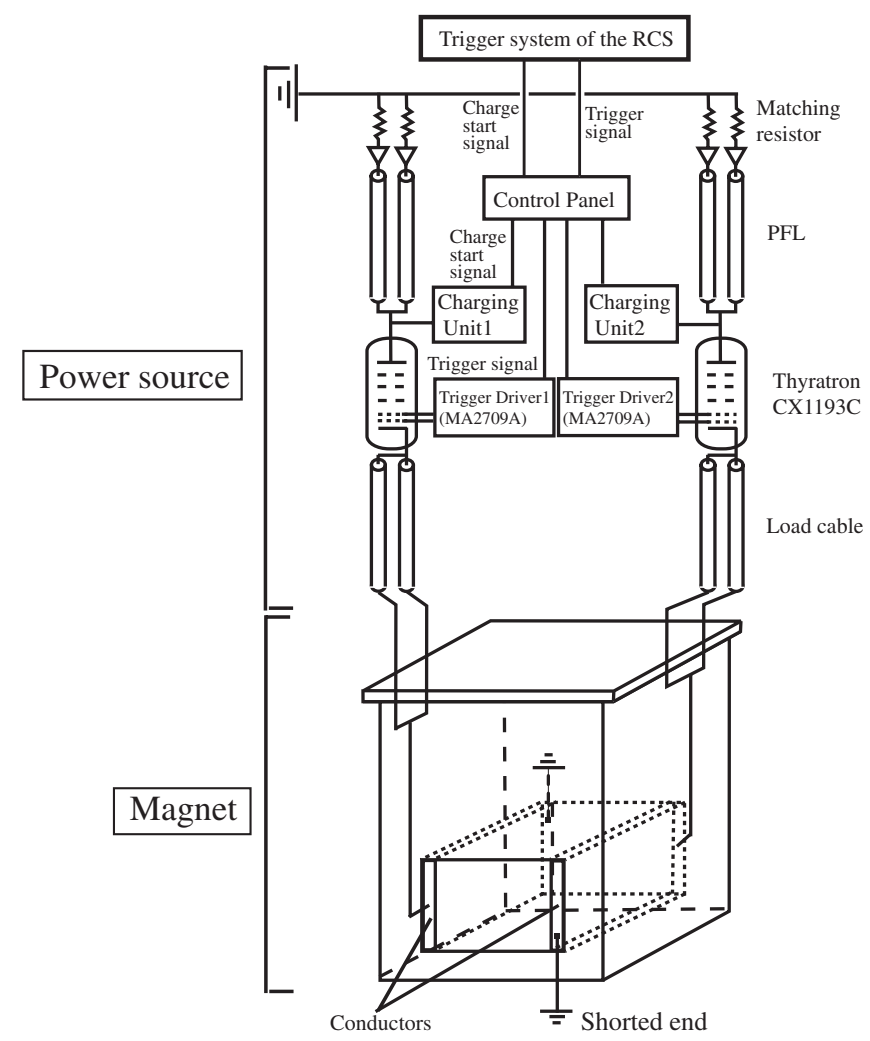

FIG. 1. Outline structure of the kicker system.

magnet. The kicker magnets are placed in order of L, M, S, (Q), S, S, M, L, and L. Figure 1 shows the outline structure of one kicker system. A kicker system consists of a power source and a magnet.

\section{B. Power supply}

One power source has an operation unit and two identical power supply units. The power supply unit is composed of a charging unit, two pulse forming lines (PFL), two matching load resistors, a thyratron, and two load cables. The H-bridge type insulated gate bipolar transistors (IGBT) are used for the switching circuit in the charging unit. Figure 2 shows the wave pattern of the charging voltage. By using the IGBT, it is possible to select the arbitrary time pattern of the charging voltage, which is called the command-charging feature. The charging time is set to $30 \mathrm{~ms}$ and the hold time is defined as $6 \mathrm{~ms}$ at the maximum charging voltage of $80 \mathrm{kV}$. If the trigger signal does not come within $37 \mathrm{~ms}$ from the start of charge, the power source automatically makes the thyratrons fire by the internal trigger. The PFL and load cables are high voltage coaxial cables, which are specially developed and manufactured for this project. The measured characteristic impedance of the coaxial cable was $19.5 \Omega$ at $20 \mathrm{MHz}$ which agreed well with the design value of $20 \Omega$. The measured capacitance and inductance per unit length of the coaxial cable was $292.5 \mathrm{pF} / \mathrm{m}$ and $0.151 \mu \mathrm{H} / \mathrm{m}$, respectively. The pulse length $\tau$ for the coaxial cable is 


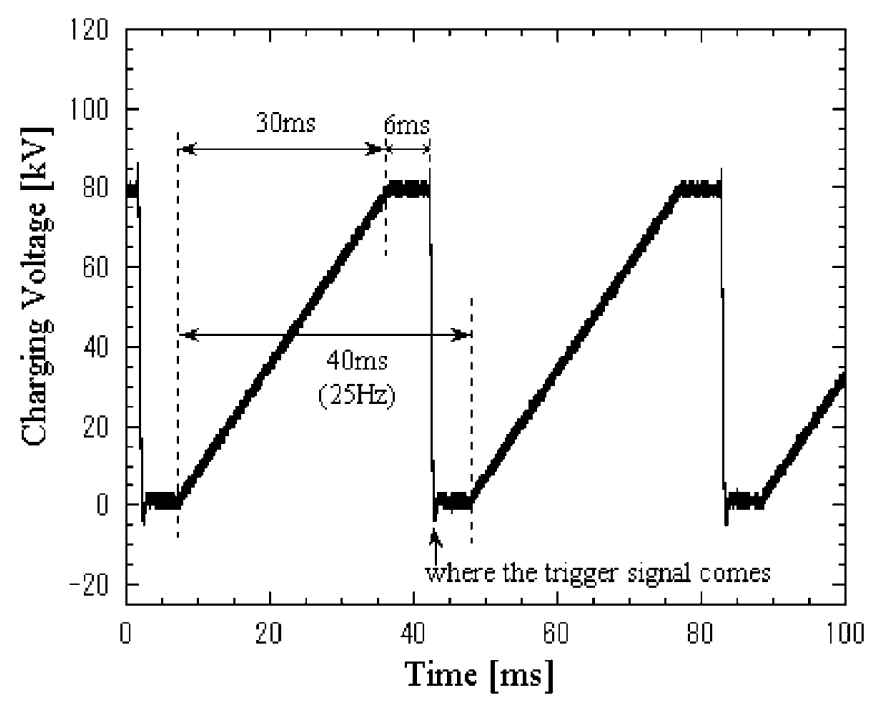

FIG. 2. Charging voltage pattern.

estimated by the formula, $\tau=2 n \sqrt{C L}$, where $n, C$, and $L$ are the length, capacitance, and inductance of the PFL. When the length of the PFL is $100 \mathrm{~m}$, the pulse length is estimated to be $\tau=1330 \mathrm{~ns}$. Although the requested flattop length is $840 \mathrm{~ns}$ in order to extract two beam bunches, the length of the PFL was selected as $102 \mathrm{~m}$ in consideration of the rise time and transmission time in the magnet. As shown in Sec. IV, this margin of the pulse length becomes very important to obtain the requested flattop characteristics. The thyratron, which is a CX1193C made by e2V Ltd., is used to switch the high voltage charged in the PFL. In total 16 thyratrons are used in the eight power sources. The comparison of the specification required by the power source and the maximum thyratron rating is shown in Table II. Because the thyratron is the key device for stable operation, a version with enough safety margin in each parameter is selected. The thyratron is mounted in a silicone oil bath for insulation and cooling. The thyratron mount structure is designed for low inductance to keep the fast pulse rise time. The trigger module MA2709A, which was made also by e2 $\mathrm{V}$ Ltd. is used to put the trigger signal to two trigger grids (G1 and G2) of the thyratron. Optical trigger signals are used for the input of MA2709A to prevent false operation due to electrical noise.

TABLE II. Comparison between the required specifications of the kicker power source and the abridged data of the thyratron CX1193C.

\begin{tabular}{lcc}
\hline \hline & Power supply Thyratron CX1193C \\
\hline Peak anode voltage & $80 \mathrm{kV}$ & $130 \mathrm{kV} \max$ \\
Peak forward anode current & $4 \mathrm{kA}$ & $10 \mathrm{kA} \max$ \\
Average anode current & $0.24 \mathrm{~A}$ & $4.0 \mathrm{~A} \mathrm{max}$ \\
Pulse repetition rate & $25 \mathrm{~Hz}$ & $100 \mathrm{~Hz}$ max \\
Rise time & $80 \mathrm{~ns}$ & $30 \mathrm{~ns}$ (typical) \\
Jitter & $10 \mathrm{~ns}$ & $5 \mathrm{~ns}$ (typical) \\
\hline \hline
\end{tabular}

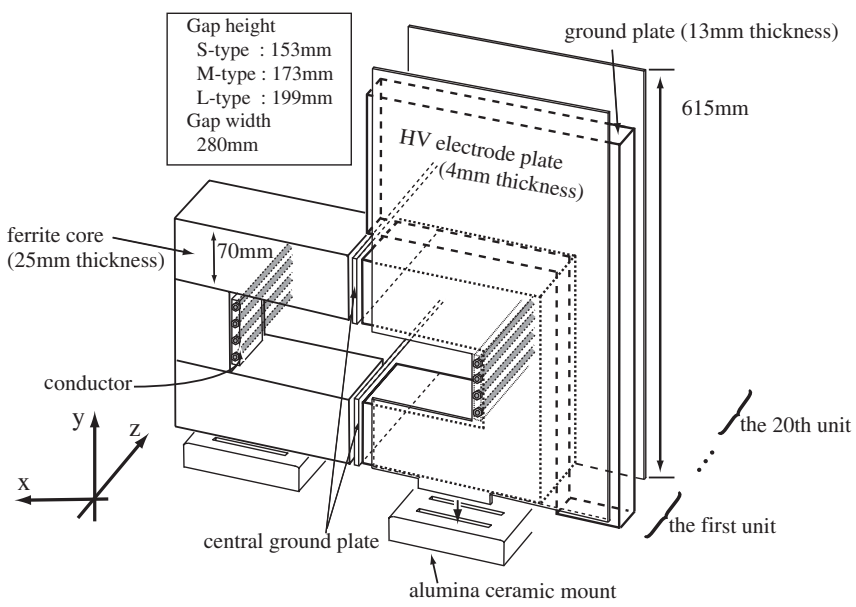

FIG. 3. Structure of the kicker magnet. The definition of each axis is also shown.

\section{Kicker magnet}

The kicker magnet of RCS is a distributed parameter type in order to obtain a fast rise time. Figure 3 shows the outline structure of a kicker magnet. Ni-Zn ferrite, PE14 made by TDK Ltd., is used as the magnetic core because of its small magnetic loss up to high frequencies. Its initial permeability remains almost constant up to $5 \mathrm{MHz}$. Aluminum alloy, A5052, is used for high voltage electrode plates, ground plates, and conductors for the excitation current. These aluminum parts were electrically polished by pit-free electropolishing, in order to reduce outgassing from the surface [8]. The ferrites are put together as Cshape cores facing each other. Each ferrite core and corresponding aluminum ground plate are inserted between the aluminum high voltage (HV) electrode plates. The conductors are also sandwiched between the HV electrode plates as shown in Fig. 3. This forms one unit of the distributed parameter circuit. In total there are 20 units. The capacitance of one unit is determined by area and distance between the ground plate and HV electrode plates, while the inductance is determined by the gap height of the magnet. They were designed for a characteristic impedance of $10 \Omega$. The measured capacitance and inductance were $404 \mathrm{pF} /$ unit and $39 \mathrm{nH} /$ unit, respectively. Therefore the measured characteristic impedance of the magnet was estimated to be $9.8 \Omega$. The end of the magnet is shortened to ground to obtain twice the excitation current because of the superposition of the forward and backward current. The current at each side of the conductors proceeds in the opposite direction generating the magnetic field. The advantage for generating the magnetic field from opposite ends is that exactly the same two units can be used in the power supply. The whole magnet is installed in the vacuum chamber in order to prevent electrical discharge on the surface of the materials. However, discharge traces had been found at the corner of the HV electrode plates and the surface of the ferrite cores after a whole day excitation test 
of the magnet at full rating. As a result of the investigation, it had been found that baking in vacuum was necessary to reduce the outgassing from the components to the level of suppressing discharge. The conditioning by continuous excitation test was very effective to achieve a stable vacuum pressure during the current excitation [9].

\section{Operational cycle}

Here, we explain one cycle of the charge and excitation process. At first the charging unit starts to charge the PFL when it receives the charge start signal. The charging process completes in $30 \mathrm{~ms}$ as shown in Fig. 2. After $36 \mathrm{~ms}$ from charge start, the trigger signal is sent to the trigger module MA2709A, which then fires the thyratron. The upper graph in Fig. 4 shows the waveform of the output current. The current rise time is typically $25 \mathrm{~ns}$ from $10 \%$ to $90 \%$. The output current goes through the $130 \mathrm{~m}$ load cable for $770 \mathrm{~ns}$. The current propagates along the conductors in the kicker magnet and excites the magnetic field. It takes $95 \mathrm{~ns}$ to propagate each way through the magnet. The lower graph in Fig. 4 shows the integrated magnetic field shape. The rise time becomes about $260 \mathrm{~ns}$ due to the propagation time through the cable and the kicker magnet itself. The current is totally reflected at the shortened end of the magnet and returns to the power source through the magnet and the load cables. Because the thyratron recovery time, which means the time required after cessation of forward anode current for the grid to regain control, is a few microseconds, the reflected current
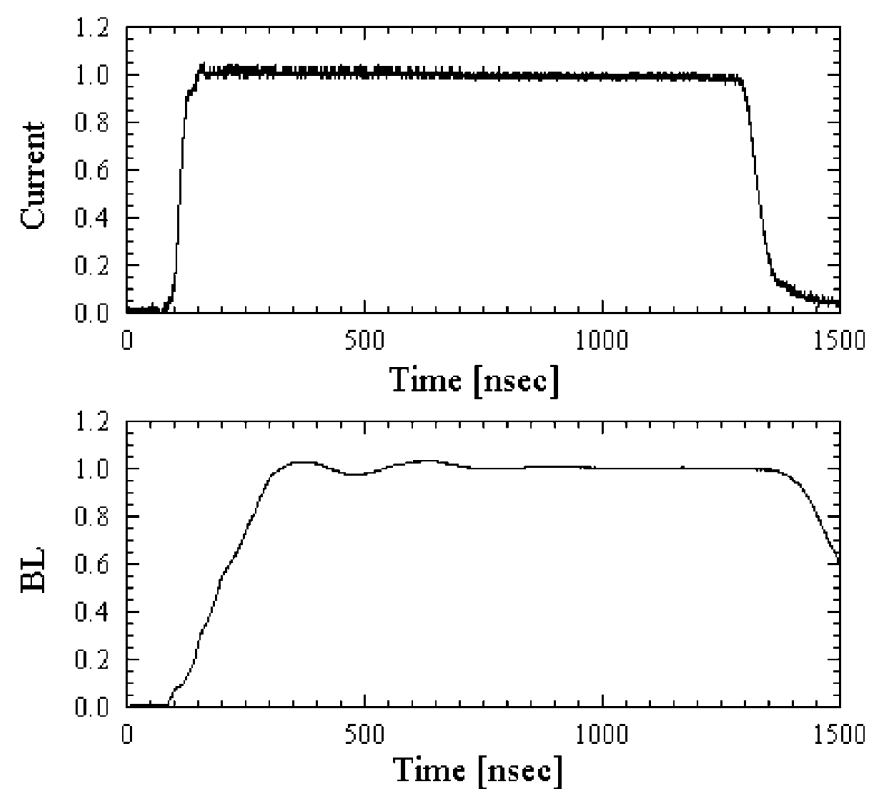

FIG. 4. Output current and integrated magnetic field, which are normalized by the average value of the flattop. The upper graph shows the output current measured by the current monitor, Pearson Electronics, Inc. model 110, which is installed at a load cable. The lower graph shows the integrated magnetic field measured by the search coil. goes through the thyratron to the PFL. Then the reflected current is finally consumed in the matching resistors at the end of the PFL.

\section{MAGNETIC FIELD}

The magnetic field, which affects the beam, is the perpendicular field component integrated along the beam path ( $z$ axis), which is called the $B L$ value. Our kicker magnet has a large gap height so that the leakage field is large at its edge. Therefore it is difficult for the $B L$ distribution along the transverse direction to obtain a wide flattop range. Using the prototype kicker magnet, we optimized the length of the coil height to obtain a better $B L$ distribution by using the fact that the magnetic field from the side of the ferrite core becomes larger when the coil height becomes shorter [7]. This result was reflected in the design of the production version of the kicker magnets. The coil height of the production versions was optimized to $83 \%$ of the gap height from the first design of $97 \%$ of the gap height. The $B L$ distribution was estimated from the results of the magnetic field mapping by using a short search coil. The measurement was performed in the aperture as widely as possible. Only the result of the S-type magnets were reported in Ref. [10]. After that, the measurement of the $B L$ distributions had been performed for all the eight magnets. In this article, we show the results of the $B L$ distributions for all the magnets. The definition of each axis in the following description is shown in Fig. 3. The origin of each axis is defined as the center of the magnet aperture.

Table III shows the measured absolute $B L$ values at $x=$ 0 for each magnet. Figure 5 shows the $B L$ distribution along the $x$ axis at $y=0$. The $\pm 0.2 \%$ error bars, which are estimated below, are shown in Fig. 5. The $B L$ values in Table III and Fig. 5 were those estimated corresponding to $60 \mathrm{kV}$ charging voltage. The $B L$ values averaged by the same type are also shown in Table III. The discrepancy in the same type is within $2.5 \%$. These discrepancies are compensated by the individual power supply current. Figure 6 shows the contour maps of the $B L$ distribution. All $B L$ values are normalized by the value at the aperture center for each magnet. The possible origins of the errors

TABLE III. Measured absolute $B L$ values at $x=0 . B L_{\text {ave }}$ is the averaged $B L$ value for the same type of the magnet.

\begin{tabular}{lrccc}
\hline \hline Type & Number & $B L[\mathrm{Tm}]$ & $B L_{\text {ave }}[\mathrm{Tm}]$ & $B L / B L_{\text {ave }}$ \\
\hline S-type & No. 3 & 0.0308 & 0.0306 & 1.006 \\
& 4 & 0.0305 & & 0.995 \\
& 5 & 0.0306 & & 0.998 \\
\hline M-type & No. 2 & 0.0277 & 0.0274 & 1.010 \\
& 6 & 0.0271 & & 0.990 \\
\hline L-type & No. 1 & 0.0246 & 0.0242 & 1.015 \\
& 7 & 0.0244 & & 1.008 \\
& 8 & 0.0236 & & 0.977 \\
\hline \hline
\end{tabular}



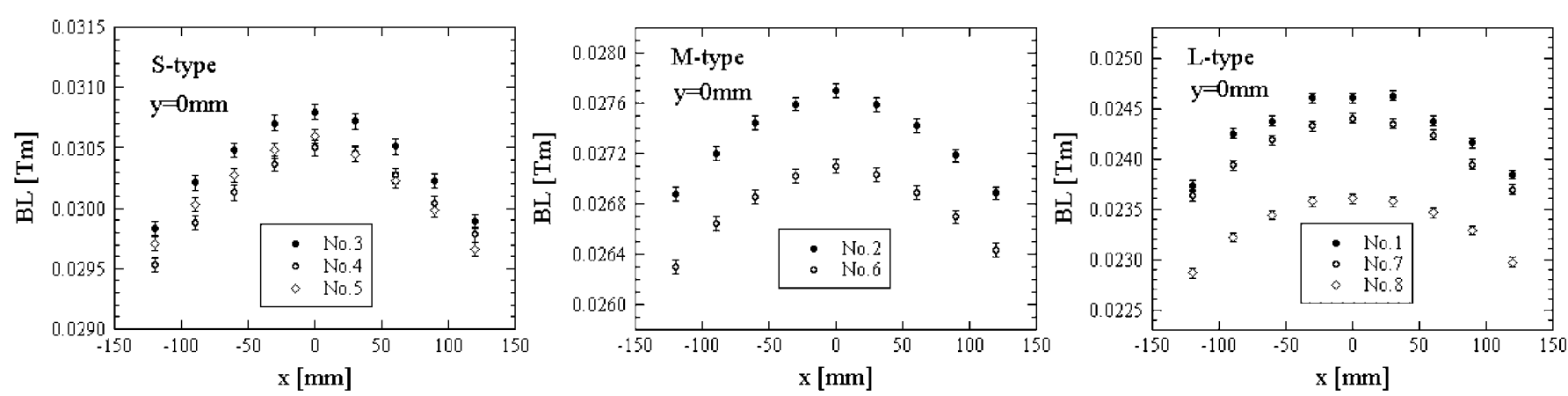

FIG. 5. The integrated magnetic field distribution for each magnet type at $y=0$. The definition of each axis is shown in Fig. 3 . Numbers 1 to 8 in the graphs refer to the magnet number described in Table I. Each data point has a $\pm 0.2 \%$ error bar.

come from the misalignment of the measurement setup, irreproducibility of the excitation current, and the measurement interval along the $z$ axis. The position of the search coil was aligned within the accuracy of $0.2 \mathrm{~mm}$ in the measurement range [10]. Therefore, the error of the $B L$ value which originated from the misalignment is negli- gible. The pulse-by-pulse irreproducibility of the excitation current was $\pm 0.3 \%$ in the case of the prototype power supply, which was used for the measurement. Therefore the error of the magnetic field was also $\pm 0.3 \%$. The error of the $B L$ value, which originates from this is estimated in the following. The integrated magnetic field $B L$ was calcu-
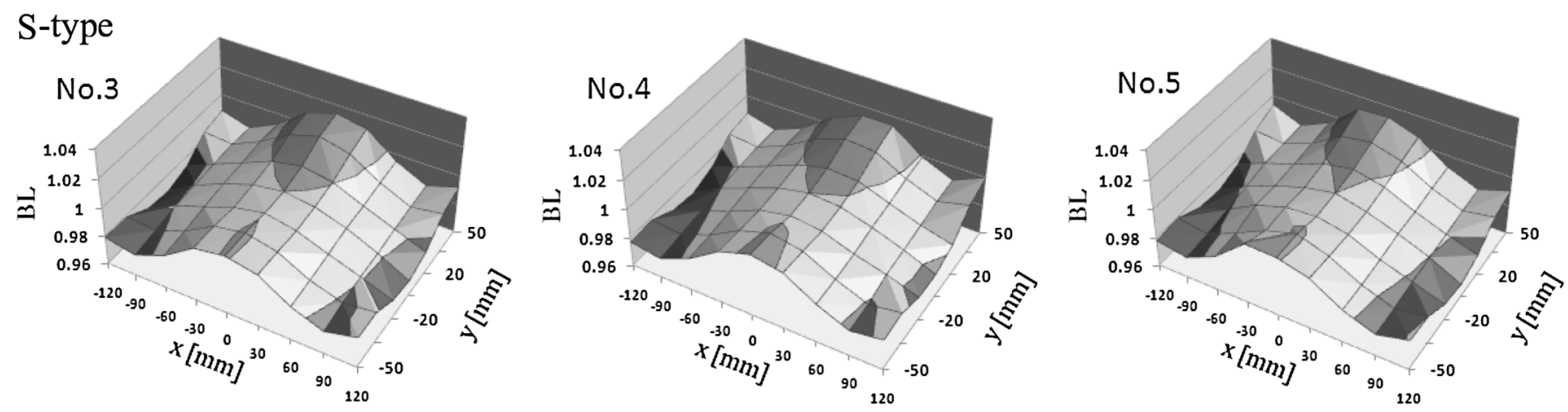

M-type
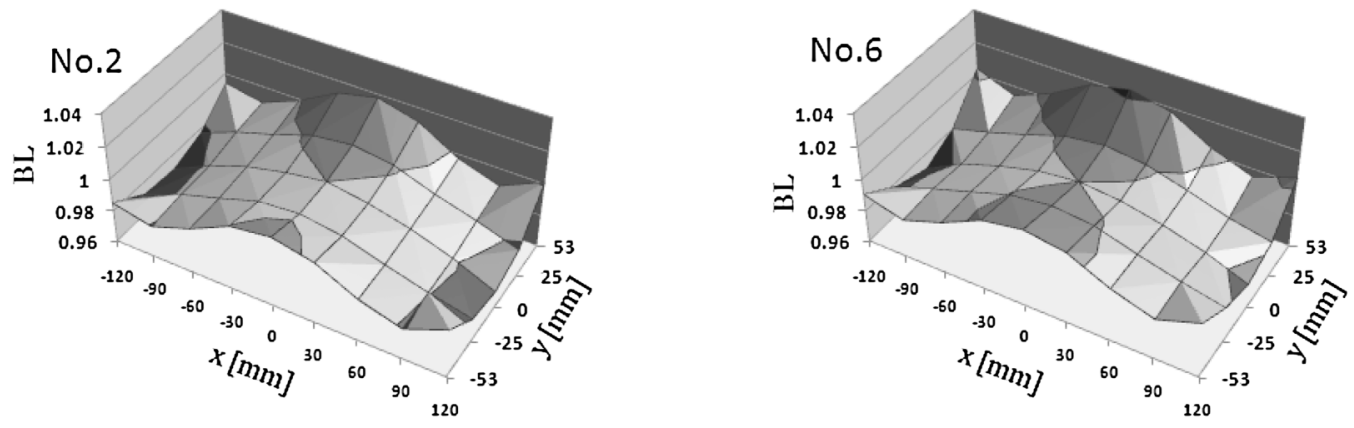

L-type
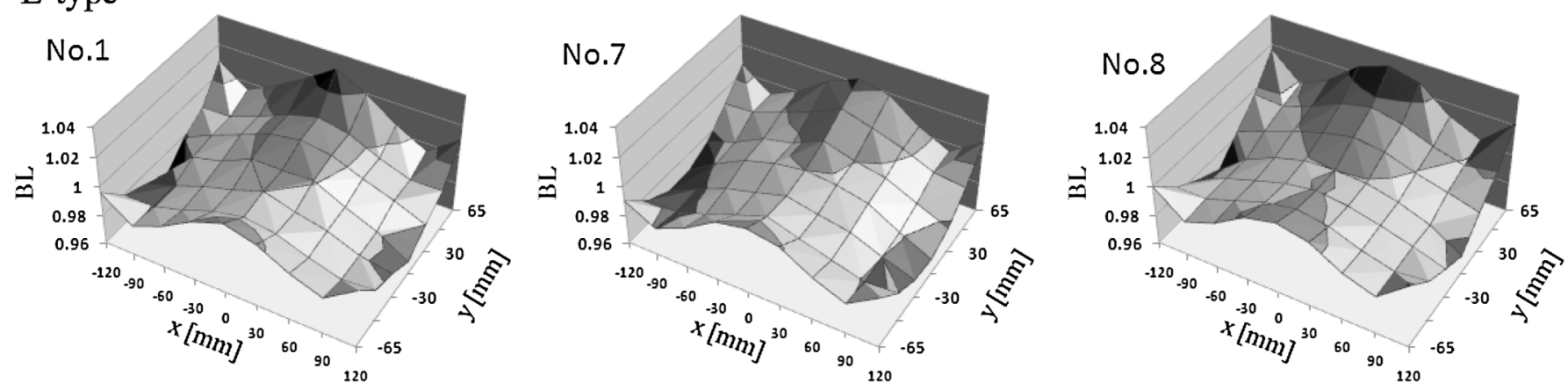

FIG. 6. The contour maps of the integrated magnetic field distribution. The definition of each axis and magnet number is the same as that of Fig. 5. 
lated using the formula,

$$
B L=\sum_{i=1}^{n} \frac{B_{i}+B_{i+1}}{2} \Delta z,
$$

where the $B_{i}$ is the magnetic field measured by the search coil at $i$ th point along the $z$ axis. The $\Delta z$, which is $40 \mathrm{~mm}$ in this measurement, is the interval of the measurement step for the $B_{i}$. The number of the measurement steps $n$ is 29 in this measurement. Ignoring $B_{1}$ and $B_{n+1}$, because these boundary points contribute only half, the error of the integrated magnetic field $\delta B L$ is estimated from Eq. (1) by using the error of the magnetic field $\delta B_{i}$ as

$$
\delta B L=\Delta z \sqrt{\sum_{i=2}^{n}\left(\delta B_{i}\right)^{2}} .
$$

Assuming $B_{i}=B$ (constant value) about $i=1-n$ for simplicity, the following relation is induced from Eqs. (1) and (2) as

$$
\frac{\delta B L}{B L}=\frac{1}{\sqrt{n-1}} \frac{\delta B}{B} .
$$

Now, the error of the magnetic field $\delta B / B$ and number of steps $n$ is $\pm 0.3 \%$ and 29, respectively, the error ratio of the integrated magnetic field $\delta B L / B L$ is $\pm 0.06 \%$. The value of $\Delta z$ which is the measurement interval along the $z$ axis should be enough small to obtain the $B L$ value which is not affected by the $\Delta z$. In order to shorten the measurement time, however, the $\Delta z$ is desired to be large. We measured the $B L$ in the interval of both 20 and $40 \mathrm{~mm}$ along a certain $z$ axis. The results were the same values within $\pm 0.1 \%$. Therefore the value of $\Delta z$ was chosen to be $40 \mathrm{~mm}$. For the error sources stated above, all the $B L$ values have an error of at least $\pm 0.16 \%$. As shown in Fig. 6, for the same magnet types the $B L$ distribution is in good agreement. The differences are relatively larger in the points distant from the center. These are the points where the leakage field from the edge and the side of the ferrite cores are large. Therefore the small misalignment of the search coil causes those differences. From the viewpoint of the beam optics, the undistorted beam can be extracted through the measured $B L$ distributions.

\section{BEAM BASED MEASUREMENT OF THE FLATTOP}

The flattop uniformity of the kicker magnetic field directly affects the profile and position of the extracted beam. The required flatness of the flattop is $2 \%$ in the time length of $840 \mathrm{~ns}$ in order to extract the two beam bunches. It was found that the disturbance of the flattop is caused by two reasons [6]. One is the impedance mismatch between load cables and corresponding magnet. The other is the effect of the magnetic field induced by the penetrated magnetic flux from the opposite side of the ferrite core. As a result, the

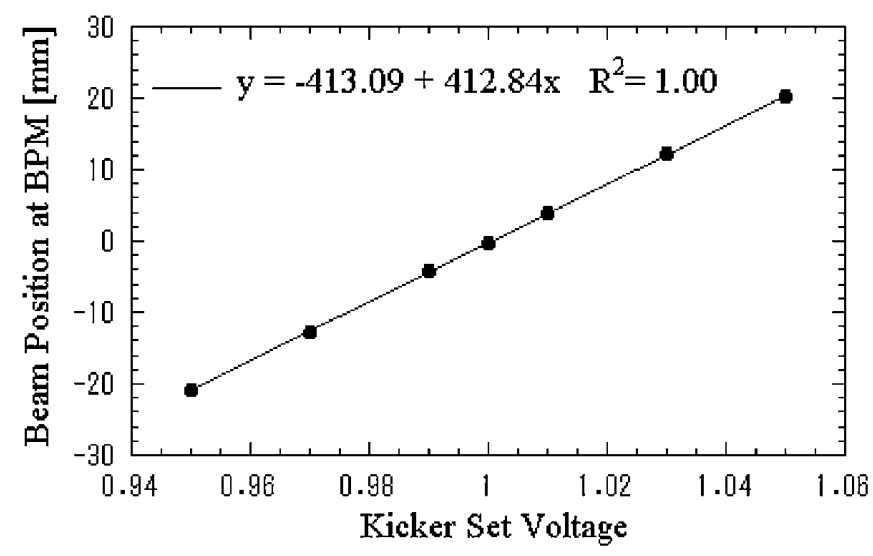

FIG. 7. The beam position shift corresponding to the kicker set voltage which is normalized by the normal value.

flattop of the kicker magnetic field showed a ringing structure as displayed by the lower graph in Fig. 4. The degree of flatness distortion which was measured by the search coil was $6 \%$, while the requested value was $2 \%$. However, there was a comment that the detail of the pulse shape measured by a search coil was less reliable, because it depended on the circuit constant of the measurement system. Therefore we decided to measure the degree of flatness by using the extracted beam itself.

Figure 7 shows the beam position shift corresponding to the set value for the kicker power supply. The beam position was measured by the beam position monitor installed at the downstream beam line. The relation was linear as expected. Using this relationship, the degree of magnetic field flatness was measured as the amount of beam position shift when the trigger timing for the kicker pulse was changed step by step. In this measurement, the longitudinal beam width was chopped to be very short, about $25 \mathrm{~ns}$ in full width at the extraction timing, in order to get better resolution. The measured magnetic field flattop is shown in the upper graph of Fig. 8. The value is normalized by the average flattop value whose time range corresponds to the requirement of two beam bunches. The result measured by search coil is also shown for comparison. The two measurement results are well consistent. Therefore we can conclude that the search coil measurement also had good accuracy. The degree of distortion was actually $6 \%$ in total. This value is larger than the required value of $2 \%$ as described above. In order to decrease the distortion, the timing adjustment of the each kicker magnet was attempted. The timing of four out of eight kickers was modified to cancel out the peaks and valleys of the flattop. The trigger timing of Nos. 2, 4, 6, and 8 kickers was delayed for about $130 \mathrm{~ns}$. The lower panel of Fig. 8 shows the results. The flatness of $2 \%$ was achieved in the time length of $850 \mathrm{~ns}$, which satisfies the requirement. Inevitable disadvantage of this timing adjustment technique is that the rise time of the magnetic field becomes larger. The rise time after timing adjustment was estimated 

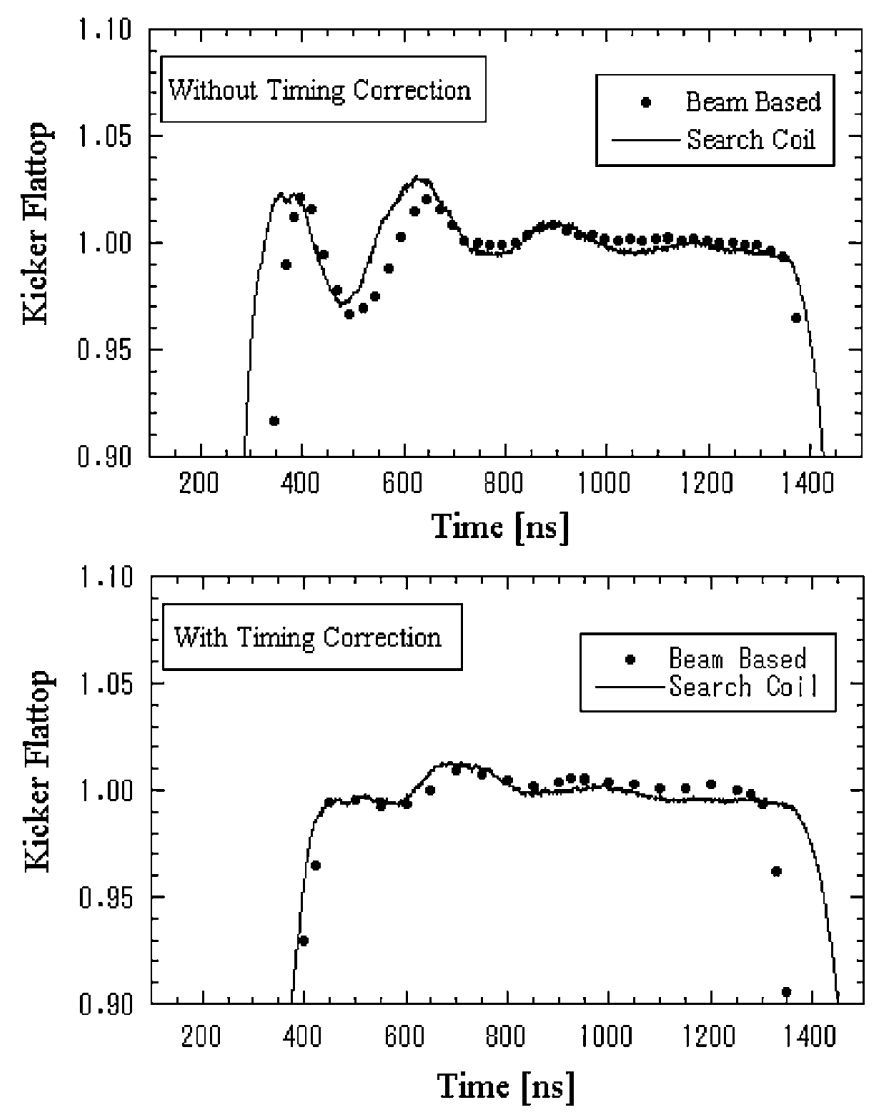

FIG. 8. Kicker flattop measured by the beam position (closed circles) and the search coil (solid line). The upper graph shows the results without timing correction, while the lower shows the results with timing correction.

by using the results with the search coil measurement. It was $340 \mathrm{~ns}$, which is acceptable because it is smaller than the time of 357 ns between two beam bunches.

\section{STABLE OPERATION}

The reproducibility of the output current of the kicker magnet determines the stability of the extracted beam orbit. It has been checked by calculation that the beam position at the exit of the extraction septum magnet changes by about $0.6 \mathrm{~mm}$ if the magnetic field of a kicker magnet changes by $1 \%$. Figure 9 shows the time dependence of the output current of the kicker power supplies. The upper panel shows the stability during one hour, while the lower panel shows the stability during a longer time range of eight hours. The fluctuation is about $0.2 \%$, which is small enough to deliver the steady beam to the downstream line.

As mentioned in the section before, 16 thyratrons are used as a switching device. Such a thyratron tube is filled with deuterium gas. The thyratron commutation is achieved by making a plasma in the region between the grid and the anode [11]. The gas pressure is the most important factor for reducing commutation time, which
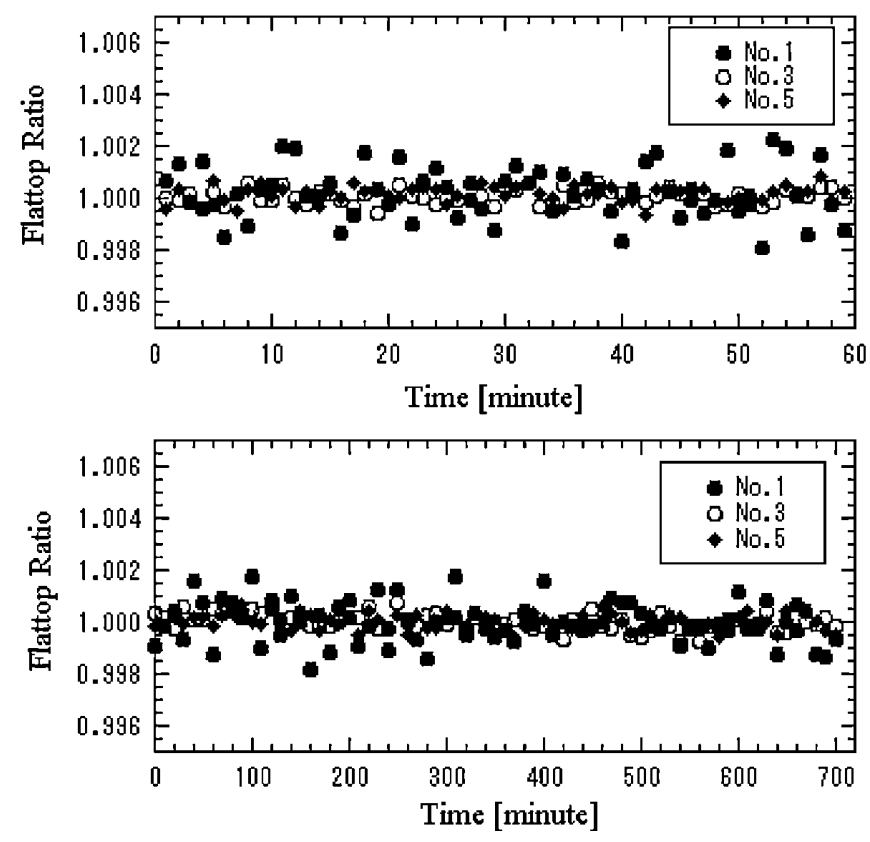

FIG. 9. Stability of the output current during one hour (the upper panel) and eight hours (the lower panel).

corresponds to the rise time of the output current for exciting the kicker magnet. Titanium hydride is used for the thyratron reservoir system. The gas pressure is maintained by the thyratron reservoir heater, which is a tungsten filament. In our operation, first, each reservoir voltage was set at the value written in the data sheet which was about 4.0-4.2 V. The rise time of several thyratrons had gradually become larger. The reservoir voltage had been changed a few times in the case of such thyratrons during the initial 2000 hours of the operation in order to keep the rise time smaller than $35 \mathrm{~ns}$. However, several thyratrons had become disabled after about 500-2000 hours operation. The inside of one of the dead tubes was investigated. We found that its cathode surface was heavily damaged by an arc discharge. A possible reason is that the gas pressure was too low. Therefore, we evaluated the reservoir voltage where the thyratron began to prefire spontaneously. Figure 10 shows the measured dependence of the rise time for four typical thyratrons on the reservoir voltage up to the spontaneous breakdown. The dependences of jitter and anode delay time, which is the time between the trigger pulse and output current, are also shown. We changed the reservoir voltage in $0.1 \mathrm{~V}$ steps and waited 5 minutes at each reservoir voltage level to allow the gas pressure to stabilize at the new level. When the spontaneous breakdown occurred within 5 minutes, it was regarded as the top limit of the reservoir voltage, $V_{\max }$. As shown in Fig. 10, the rise time and anode delay time of S/N 1941 and 1946 show smooth characteristic curves. Their jitter shows small values of about 5 ns. However, S/N 1904 and 1932 show a peculiar characteristic. Their characteristic curves of the rise time and anode delay time are not 

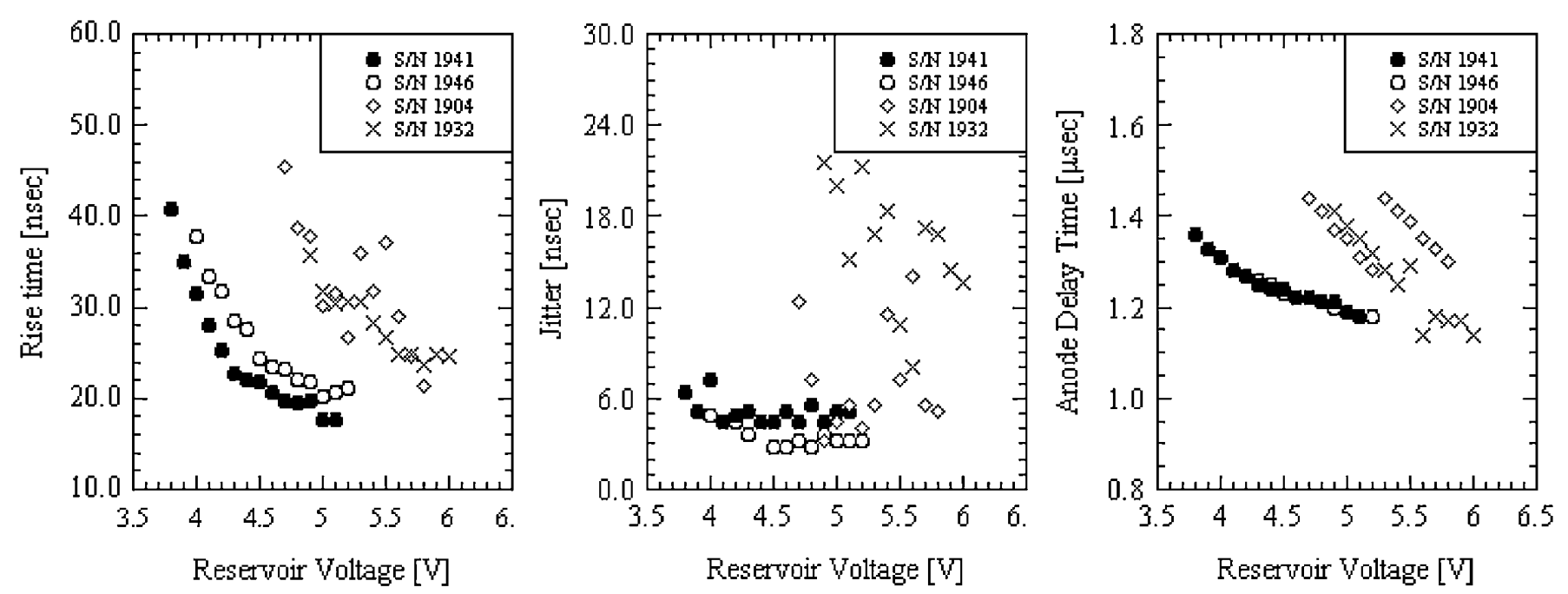

FIG. 10. Dependence of rise time, jitter, and anode delay time on reservoir voltage.

smooth. The jitter is more than 10 ns. Furthermore, the reservoir voltage must be set to a value much higher than recommended in the data sheet, which was $4.0-4.2 \mathrm{~V}$, to obtain the fast rise time. The data in Fig. 10 was measured after about 2000 hours operation. Eventually, these two tubes, S/N 1904 and 1932, became disabled after about 2400 hours operation. This is attributed to the fact that these tubes' cathode had been damaged during the initial operation with low reservoir voltage and this reduced the tube life. The reservoir voltage dependence was measured for all thyratrons up to the $V_{\max }$. Then the reservoir voltage was set to $V_{\max }-0.2 \mathrm{~V}$ for the operation. When the prefire occurred more than once a day even after that adjustment, the reservoir voltage was reduced by another $0.1 \mathrm{~V}$. After the adjustment, the number of prefires of most thyratrons was reduced to less than 5 during two weeks operation. However, there are a few tubes which are easy to prefire spontaneously even after the reservoir voltage adjustment. These tubes broke down more than 10 times during the two weeks operation. We plan to replace these tubes with new ones. As shown in Fig. 10, when the reservoir voltage is changed, the delay time between the trigger signals to the output current also changes because the delay time largely depends on the gas pressure in the tube. The delay time can be adjusted by delay modules in the power supplies, so that after firing all output currents appear at the right time. Although there is no data for the lifetime of this type of tube when it is operated under optimal gas pressure, the maker indicates that it would be about 5000 hours in the case of $25 \mathrm{~Hz}$ operation. Therefore, each thyratron may have to be replaced with a new one once per year.

\section{SUMMARY}

The kicker magnets in J-PARC RCS succeeded in extracting the proton beams and have been operated stably from the first commissioning with beam in October 2007. The kicker system specification is very challenging be- cause it aims to extract the $1 \mathrm{MW}$ proton beams. The magnetic field measurement had been precisely performed for all kicker magnets. The distribution of the integrated magnetic field was flat enough to kick the $486 \pi \mathrm{mm}$ mrad beam without distortion. The flattop shape of the magnetic field was measured by the beam based method. The distortion of the flattop was found to be $6 \%$, which exceeds the specified limit of $1 \%$. The timing adjustment was performed to cancel out the flattop distortion. As a result, the flatness of better than $1 \%$ was achieved. The fluctuation of the output current flattop was about $0.2 \%$, which was enough small for the steady beam. Dependence of rise time, jitter, and anode delay time on the thyratron reservoir voltage was rigorously measured. It was found that the damaged thyratrons were distinguished by the strict measurement of the dependence on the reservoir voltage. After adjustment of the reservoir voltages, the number of selfbreakdowns could be reduced.

\section{ACKNOWLEDGMENTS}

We gratefully acknowledge the people of the manufacturer IDX Co., Ltd., Tokin Machinery Corporation, involved in the challenging construction of this kicker system. We would like to thank Dr. R. Sheldrake of e2V for his expert advice on the selected thyratron. Finally, we appreciate the support of all RCS staff members during the experiment and beam commissioning.

[1] High-intensity Proton Accelerator Project Team, J-PARC Report No. JAERI-Tech 2003-044, 2003.

[2] F. Tamura, A. Schnase, and M. Yoshii, Phys. Rev. ST Accel. Beams 11, 072001 (2008).

[3] H. Matsumoto, J.S. Oh, W. Namkung, and H. Urakata, Proceedings of LINAC 2006 (JACoW, Knoxville, Tennessee, 2006), p. 622.

[4] G. Nassibian, IEEE Trans. Nucl. Sci. 26, 4018 (1979). 
[5] M. J. Barnes, G. D. Wait, and I. M. Wilson, Proceedings of the EPAC'94, p. 2547 (1994).

[6] J. Kamiya, T. Takayanagi, T. Kawakubo, S. Murasugi, and E. Nakamura, IEEE Trans. Appl. Supercond. 16, 168 (2006).

[7] J. Kamiya, T. Ueno, and T. Takayanagi, IEEE Trans. Appl. Supercond. 16, 1362 (2006).

[8] K. Tajiri, Y. Saito, Y. Yamanaka, and Z. Kabeya, J. Vac. Sci. Technol. A 16, 1196 (1998).

[9] J. Kamiya, M. Kinsho, N. Ogiwara, M. Kuramochi, T.
Ueno, T. Takayanagi, O. Takeda, M. Watanabe, Y. Yamazaki, and M. Yoshimoto, J. Vac. Soc. Jpn. 50, 371 (2007).

[10] J. Kamiya, M. Kinsho, M. Kuramochi, T. Takayanagi, O. Takeda, T. Ueno, M. Watanabe, K. Yamamoto, Y. Yamazaki, and M. Yoshimoto, IEEE Trans. Appl. Supercond. 18, 293 (2008).

[11] E2VTechnologies, A1A-Hydrogen Thyratrons Preamble Issue 3 (2002). 